\title{
Nature-inspired chiral metasurfaces for circular polarization detection and full-Stokes polarimetric measurements
}

\author{
Ali Basiri $\mathbb{1}^{1,2}$, Xiahui Chen ${ }^{1,2}$, Jing Bai ${ }^{1,2}$, Pouya Amrollahi $\mathbb{1}^{3}$, Joe Carpenter $\mathbb{D}^{1,2}$, Zachary Holman ${ }^{1,2}$, \\ Chao Wang $\mathbb{B}^{1,2,3}$ and Yu Yao (1) ${ }^{1,2}$
}

\begin{abstract}
The manipulation and characterization of light polarization states are essential for many applications in quantum communication and computing, spectroscopy, bioinspired navigation, and imaging. Chiral metamaterials and metasurfaces facilitate ultracompact devices for circularly polarized light generation, manipulation, and detection. Herein, we report bioinspired chiral metasurfaces with both strong chiral optical effects and low insertion loss. We experimentally demonstrated submicron-thick circularly polarized light filters with peak extinction ratios up to 35 and maximum transmission efficiencies close to $80 \%$ at near-infrared wavelengths (the best operational wavelengths can be engineered in the range of 1.3-1.6 $\mu \mathrm{m}$ ). We also monolithically integrated the microscale circular polarization filters with linear polarization filters to perform full-Stokes polarimetric measurements of light with arbitrary polarization states. With the advantages of easy on-chip integration, ultracompact footprints, scalability, and broad wavelength coverage, our designs hold great promise for facilitating chip-integrated polarimeters and polarimetric imaging systems for quantum-based optical computing and information processing, circular dichroism spectroscopy, biomedical diagnosis, and remote sensing applications.
\end{abstract}

\section{Introduction}

Circularly polarized light (CPL) has been widely used in quantum communication ${ }^{1}$, quantum computing ${ }^{2,3}$, circular dichroism (CD) spectroscopy ${ }^{4}$, and polarimetric imaging and sensing ${ }^{5-7}$. Traditionally, CP light detection requires multiple bulky optical elements such as polarizers, waveplates, and mechanically rotating components ${ }^{8}$, which poses fundamental limitations for device miniaturization, robust system integration, and high-speed operation. Organic chiral molecules have been proposed for miniaturization of CPL detection devices, such as liquid crystals (LCs ${ }^{9,10}$, chiral dyes ${ }^{11}$, and helicene-based

\footnotetext{
Correspondence: Chao Wang (wangch@asu.edu) or Yu Yao (yuyao@asu.edu) ${ }^{1}$ School of Electrical, Computer and Energy Engineering, Arizona State University, Tempe, AZ 85281, USA

${ }^{2}$ Centre for Photonic Innovation, Arizona State University, Tempe, AZ 85281, USA

Full list of author information is available at the end of the article.
}

chiral semiconductor transistors ${ }^{12}$. Recent developments in nanotechnology and nanophotonics have enabled ultracompact solid-state CPL detection ${ }^{13-23}$ (see Supplementary Table S1). Compared with organic chiral molecules, nanostructure-based devices generally exhibit superior stability in ambient conditions, fast response time, and high fidelity. For example, artificial threedimensional (3D) metamaterials have been produced based on chiral L-shaped ${ }^{22}$, helical ${ }^{20,24-26}$, and spiral $^{21}$ nanostructures to differentiate the handedness of CPL. However, the fabrication of these complex 3D structures requires stringent process control, and scalability is challenging. More recently, planar (or 2D) chiral plasmonic metasurface structures composed of gammadions $^{16}$, Z-shaped antennas ${ }^{17}$, spiral slots ${ }^{18}$, and even stacks of twisted planar and achiral structures ( $\operatorname{crosses}^{14}$, nanorods $^{15,23}$, etc.) with chiro-optical responses have been reported. Compared with $3 \mathrm{D}$ chiral metamaterials, 
Table 1 Full-Stokes polarimetric imaging techniques

\begin{tabular}{|c|c|c|c|c|}
\hline Structure design & Operation mode & $\begin{array}{l}\text { Operational } \\
\text { wavelength }\end{array}$ & Efficiency & Error of Stokes parameters (average) \\
\hline Plasmonic metagrating ${ }^{34}$ & Reflection, diffraction & $700-1000$ nm & $<50 \%$ & $\sim 10 \%$ \\
\hline $\begin{array}{l}\text { Diffracting integrated plasmonic } \\
\text { metasurfaces }{ }^{35}\end{array}$ & Reflection, diffraction & $500-700 \mathrm{~nm}$ & $\begin{array}{l}36-55 \% \\
\text { (numerical) }\end{array}$ & NA \\
\hline $\begin{array}{l}\text { Graphene-integrated anisotropic } \\
\text { metasurfaces }{ }^{36}\end{array}$ & Reflection & $6.7-6.8 \mu \mathrm{m}$ & $\sim 10 \%$ & $>3.9 \%(\mathrm{~S} 1),>6.5 \%(\mathrm{~S} 2),>2.5 \%(\mathrm{~S} 3)$ \\
\hline $\begin{array}{l}\text { SPP excitation by X-shaped aperture } \\
\text { array }^{38}\end{array}$ & Transmission & $750-1050 \mathrm{~nm}$ & $<4 \%$ & $\begin{array}{l}7.3-12.3 \%(\mathrm{~S} 1), 7.2-27.4 \%(\mathrm{~S} 2), \\
5.2-17.7 \%(\mathrm{~S} 3)\end{array}$ \\
\hline Integrated plasmonic polarimeter ${ }^{39}$ & Absorption & $\sim 830 \mathrm{~nm}$ & NA & $\sim 45 \%$ \\
\hline Metasurface in-line polarimeter ${ }^{37}$ & Scattering & $1500-1565 \mathrm{~nm}$ & NA & $6 \%(\mathrm{~S} 1), 5.8 \%(\mathrm{~S} 2), 4.7 \%(\mathrm{~S} 3)$ \\
\hline $\begin{array}{l}\text { Spin-orbit interaction of light with } \\
\text { scatterers }^{50}\end{array}$ & Transmission & $1.5-1.6 \mu \mathrm{m}$ & NA & 7.4\% (S1), 15.6\% (S2), $11.4 \%$ (S3) \\
\hline Dielectric metasurface $e^{40}$ & $\begin{array}{l}\text { Transmission, } \\
\text { diffraction }\end{array}$ & $845-855 \mathrm{~nm}$ & $60-65 \%$ & $7.5-15 \%$ \\
\hline $\begin{array}{l}\text { This proposal: metal-dielectric } \\
\text { hybrid (ODLM) }\end{array}$ & Transmission & $1.4-1.55 \mu \mathrm{m}$ & $\sim 80 \%$ & $1.9 \%(\mathrm{~S} 1), 2.7 \%(\mathrm{~S} 2), 7.2 \%(\mathrm{~S} 3)$ \\
\hline
\end{tabular}

Reported errors for the Stokes parameters are the arithmetic mean (Ra) extracted from the data presented in each article

metasurface structures are easier to fabricate and more compatible with on-chip manufacturing technologies; however, the chiral plasmonic metasurfaces experimentally demonstrated so far usually suffer from low circular polarization extinction ratios (CPERs) (less than $\sim 5$ ) and limited optical efficiency in experiments $(20-50 \%)$. To improve the optical efficiency, chiral dielectric metasurface structures have been achieved in experiments ${ }^{13,19}$ with optical efficiencies as high as $90 \%$. However, the CPERs of these surfaces are still limited (up to eight). To date, the realization of ultracompact CPL detection devices with simultaneously high extinction ratios and optical efficiencies is still challenging.

In addition to CPL detection, ultracompact polarimetric detection and imaging systems are highly desirable for full polarization state measurements in various applications such as communication ${ }^{27,28}$, remote sensing ${ }^{29}$, polarization imaging ${ }^{30}$, and biological diagnostics ${ }^{31-33}$. Among different polarimeter designs (Table 1), plasmonics-based polarimetric detecting devices have been reported with unprecedented compactness based on phase-gradient birefringent metagratings ${ }^{34}$, diffracting plasmonic metasurfaces $^{35}$, and graphene-integrated anisotropic metasurfaces ${ }^{36}$. However, these devices operate in reflection mode and hence are not compatible with direct on-chip integration in photodetectors or imaging sensors. Polarization-dependent surface plasmon polariton (SPP) structures have been demonstrated with feasibility for direct integration in detectors or imaging sensors ${ }^{37-39}$; however, these devices suffer from low efficiency. Very recently, a highly efficient phase-gradient all-dielectric metasurface polarimeter ${ }^{40}$ was reported with a high efficiency $(60-65 \%)$. This approach enables splitting and focusing of light in three different polarization bases in transmission mode and provides a feasible method to realize on-chip polarimetric imaging arrays. However, the polarization measurement accuracy is fundamentally limited by the crosstalk between different polarization states and noticeably degrades as the super-pixel size becomes smaller than $7.2 \mu \mathrm{m}$.

Inspired by the compound eyes of stomatopods ${ }^{41,42}$, in this paper, we theoretically and experimentally demonstrated double-layer chiral metasurface structures for near-infrared wavelength polarimetric detection with CPERs as high as 35 and transmission efficiencies $>80 \%$. The structure consists of a low-loss dielectric metasurface, an oxide spacer layer, and a nanowire polarizer, with a total thickness of less than $1 \mu \mathrm{m}$. In addition to CPL detection units, we integrated the chiral metasurface structures on the same chip with linear polarization (LP) filters to perform full-Stokes polarimetric detection. Our designs are advantageous due to the feasibility of direct and scalable integration onto existing imaging sensors, high extinction ratios, high transmission efficiencies, ultracompact footprint (subwavelength thickness, micrometer scale in the lateral dimension), and robustness; thus, these designs are ideal for ultracompact imaging, sensing, communication, and navigation systems. 


\section{Results}

\section{Design}

In nature, stomatopods (or mantis shrimps) possess extraordinary circular polarization vision due to the unique ommatidium designs in the midband of their eyes $^{42,43}$. Each ommatidium, as shown in Fig. 1a, has a top retinular cell (R8) and seven bottom retinular cells (R1-7). The R8 cell acts as a quarter waveplate (QWP) to convert CPL to linearly polarized light (LPL). The microvilli of the R1-7 cells function as wire-grid polarizers that are oriented $45^{\circ}$ with respect to the long axis of the R8 cell to discriminate converted LPL of different orientations. Inspired by this unique natural design, we created vertically integrated double-layer metasurface designs that mimic the ommatidium of stomatopods to distinguish CPL with different handedness. The ommatidium-like double-layer metasurface (ODLM) design is composed of a nanostructured birefringent metasurface acting as a $\mathrm{QWP}^{44}$, a linear polarizing nanograting, and a dielectric spacer layer between these components, as shown in Fig. 1b. The birefringence of the metasurface is achieved with an optically isotropic material, e.g., silicon, by patterning this material into nanostructures with structural anisotropy. Importantly, although neither the top metasurface nor the bottom linear polarizer is chiral, the combination of the two materials results in a hybrid chiral metasurface structure with no inversion center or reflection symmetry. Here, we introduce an angle of $\pm 45^{\circ}$ between the fast axis of the metasurface QWP and the axis of the lower polarizing nanograting; thus, the mirror symmetry is broken in these double-layer chiral metasurface structures.
To obtain a quantitative description of the device working principles without tedious full-wave simulations, we set up a simplified model of the proposed ODLM structure based on Jones matrices and obtained the transmissions corresponding to left-handed (LCP Jones vector $\frac{\sqrt{2}}{2}\left[\begin{array}{l}1 \\ i\end{array}\right]$ ) and right-handed incident CP light (RCP $\frac{\sqrt{2}}{2}\left[\begin{array}{c}1 \\ -i\end{array}\right]$ ) (for more details, see the Supplementary Information section 1):

$$
\begin{aligned}
T_{L C P}= & \frac{1}{4}\left(\left|\gamma_{x}^{V / H} \gamma_{f} e^{-i\left(\frac{\Delta \phi}{2}+\frac{\pi}{4}\right)}+\gamma_{x}^{V / H} \gamma_{s} e^{i\left(\frac{\Delta \phi}{2}+\frac{\pi}{4}\right)}\right|^{2}\right. \\
& \left.+\left|-\gamma_{y}^{V / H} \gamma_{f} e^{-i\left(\frac{\Delta \phi}{2}+\frac{\pi}{4}\right)}+\gamma_{y}^{V / H} \gamma_{s} e^{i\left(\frac{\Delta \phi}{2}+\frac{\pi}{4}\right)}\right|^{2}\right) \\
T_{R C P}= & \frac{1}{4}\left(\left|\gamma_{x}^{V / H} \gamma_{f} e^{i\left(-\frac{\Delta \phi}{2}+\frac{\pi}{4}\right)}+\gamma_{x}^{V / H} \gamma_{s} e^{-i\left(-\frac{\Delta \phi}{2}+\frac{\pi}{4}\right)}\right|^{2}\right. \\
& \left.+\left|-\gamma_{y}^{V / H} \gamma_{f} e^{i\left(-\frac{\Delta \phi}{2}+\frac{\pi}{4}\right)}+\gamma_{y}^{V / H} \gamma_{s} e^{-i\left(-\frac{\Delta \phi}{2}+\frac{\pi}{4}\right)}\right|^{2}\right)
\end{aligned}
$$

where $\Delta \phi$ is the phase retardation introduced by the QWP (note the fast axis is oriented $-45^{\circ}$ with respect to the $x$ axis) and $\gamma_{f}$ and $\gamma_{s}$ are the transmission coefficients of the QWP for the electric field components along the fast and slow axes, respectively. $\gamma_{x}^{V / H}$ and $\gamma_{y}^{V / H}$ are the transmission coefficients of the polarizing nanograting for the electric field components along the $x$ and $y$ axes, corresponding to the vertical $(V)$ or horizontal $(H)$ nanograting orientation, respectively. We apply equation (1) to calculate the dependence of the CPER on the performance of
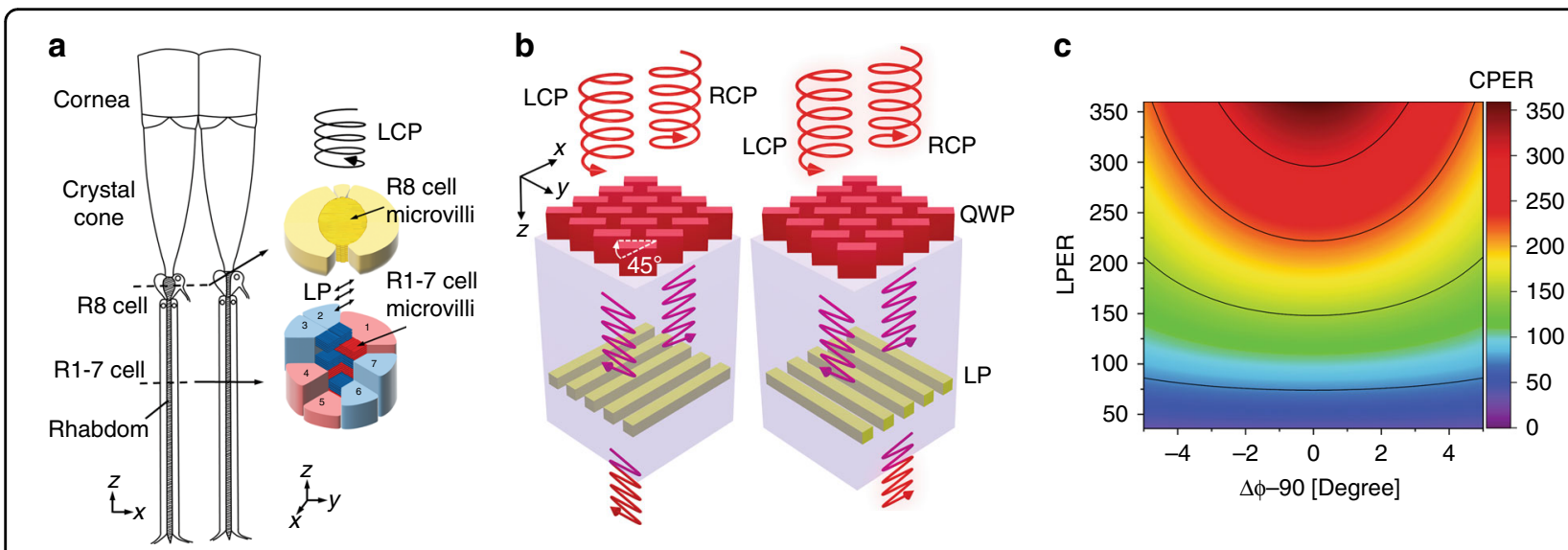

Fig. 1 Bioinspired chiral metasurface design. a Anatomical schematic of ommatidium in the compound eye of mantis shrimp responsible for circularly polarized light detection. Left side shows the longitudinal cross section of the midband in the compound eye. Right side shows the transverse cross section of the R8 and R1-7 cells along the dashed line in the longitudinal cross section. $\mathbf{b}$ Schematics of ommatidium-like doublelayer metamaterial (ODLM) design, where the dielectric metasurface behaves as artificial R8 cells and the nanogratings behave as R1-7 microvilli that differentiate the linear polarization perpendicular or parallel to the microvilli axis. c Theoretically calculated CPER based on Jones calculus, as a function of the relative phase difference between the fast and slow axes of the metasurface QWP and LPER of the polarizing nanograting. a was adapted with permission from Nature Publishing Group ${ }^{42}$ 


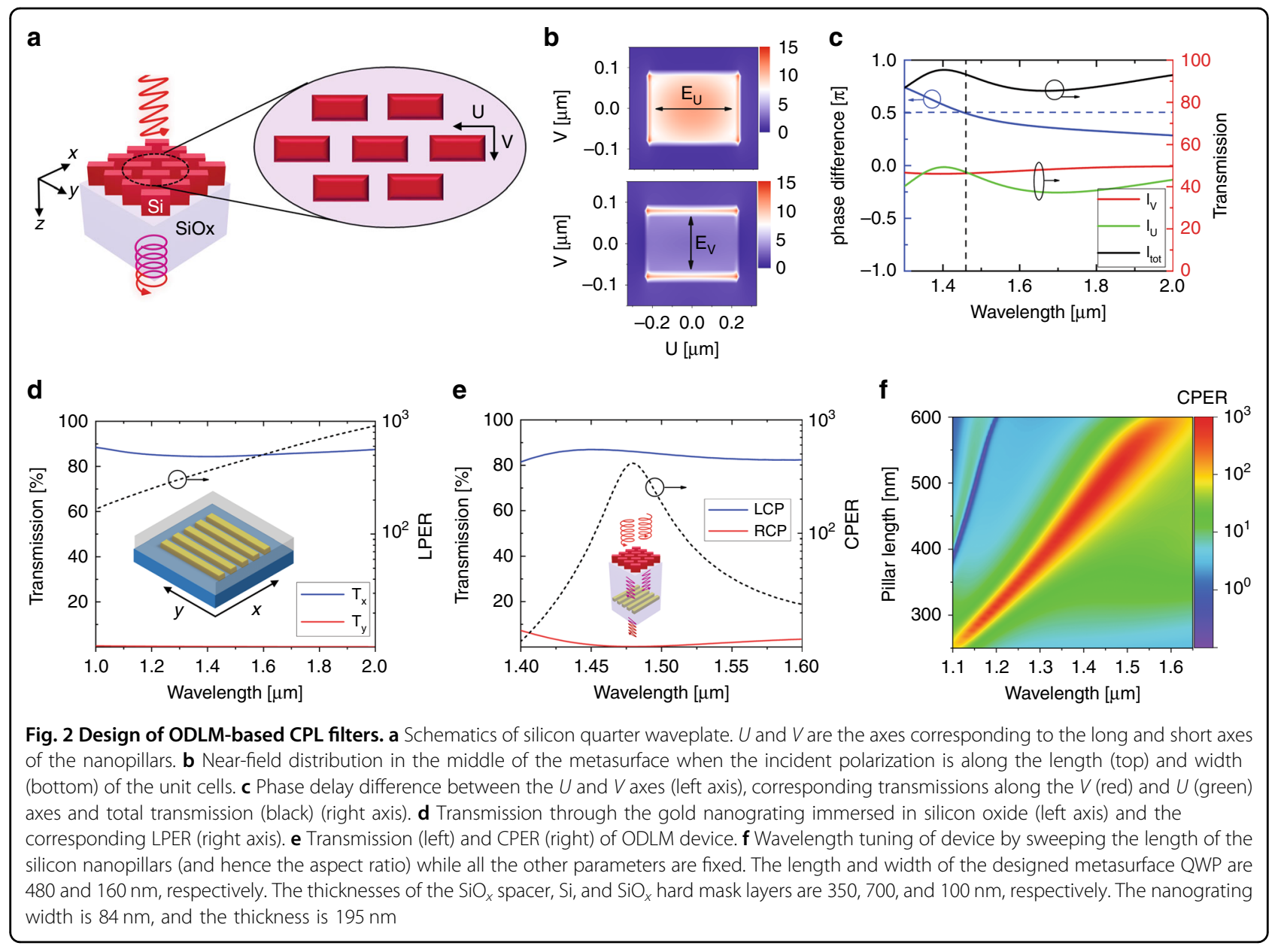

the QWP and linear polarizer, as shown in Fig. 1c. The results indicate that the maximum CPER occurs at $\Delta \phi=$ $\frac{\pi}{2}$ and is ultimately limited by the extinction ratio of the linear polarizer $\left(L P E R=\gamma_{i}^{V / H} / \gamma_{j}^{V / H}\right.$ for gratings oriented along the $j$-axis, $i, j=x, y$ ). Therefore, to achieve a high CPER, we need to maximize the LPER and design a perfect QWP with $\Delta \phi=\frac{\pi}{2}$.

We choose dielectric metasurfaces composed of silicon nanopillars with geometrically induced birefringence (Fig. 2a) to realize QWPs with accurate phase retardation control and high transmission efficiency. The Si nanopillars are designed with subwavelength dimensions (length $480 \mathrm{~nm}$, width $160 \mathrm{~nm}$, and height $700 \mathrm{~nm}$ ) to avoid Mie resonance. The birefringence of the silicon nanopillar array results from the distinct near-field distributions for different incident light polarization ${ }^{45-47}$, as illustrated in Fig. 2b. For incident LP light polarized along the slow axis ( $U$-axis) of the metasurface QWP, the electric field intensity is mostly localized in silicon (top panel in Fig. 2b). In contrast, for incident light polarized along the fast axis ( $V$-axis) of the metasurface QWP, the electric field intensity is mostly located in the air gaps between the Si pillars (bottom panel in Fig. 2b). As a result, the metasurface QWP phase retardation between the fast and slow axes can be precisely engineered by adjusting the metasurface geometry to be exactly $\pi / 2$ at the operation wavelength, e.g., at $1.47 \mu \mathrm{m}$, as shown in Fig. 2c. Moreover, the transmission coefficients are designed to be the same for the LP components along both the fast and slow axes, with a total transmission efficiency over $90 \%$ around the operation wavelength. The linear polarizer design is composed of gold nanowires with optimized dimensions (period $230 \mathrm{~nm}$, duty cycle 37\%, and thickness $195 \mathrm{~nm}$ ) to achieve an LPER greater than 200 and transmission efficiency $>85 \%$ for the wavelength range from 1 to $2 \mu \mathrm{m}$ (Fig. $2 \mathrm{~d})$. More details about the dependence of the nanograting performance on the geometric parameters is included in the Supplementary Information (Fig. S2).

Although the Jones matrix model provides an intuitive physical model to illustrate the device physics and analytical results to assist in device design, this model is too simplified to consider multiple reflections inside the thin film structure and the near-field interactions between the 
metasurface QWP and polarizing nanograting. To provide an accurate performance analysis of the ODLM-based CPL filters proposed, we performed full-wave simulations to obtain the transmission spectra of the ODLM structure for LCP and RCP incident light. Figure 2e shows the results of an ODLM-based LCP filter designed at the operation wavelength of $\lambda_{0}=1.47 \mu \mathrm{m}$. The maximum CPER is greater than 400 with a transmission efficiency greater than $85 \%$ at the operation wavelength. This design can operate over a wavelength range of more than $100 \mathrm{~nm}$ (from 1.45 to $1.55 \mu \mathrm{m}$ ) with CPER over 30 and transmission efficiency over $80 \%$. The operation wavelength $\lambda_{0}$ of the ODLM CP filters can be tailored by changing the design parameters. We obtain devices with operation wavelengths from 1.1 to $1.65 \mu \mathrm{m}$ by simply varying the length-to-width aspect ratio of the silicon pillars, as shown in Fig. 2f. The operation wavelength can also be engineered by adjusting the pillar height and the silicon volume filling factor (see Supplementary Information Fig. S1). Such engineering flexibility can enable on-chip wavelength multiplexing of the ODLM-based CPL filters with optimized device performance over broadband wavelengths.

\section{Device fabrication}

The major challenge in the fabrication of ODLM-based CPL filters is to reliably integrate metasurface QWPs and the polarizing nanograting without degrading the optical performance or affecting the overall structural stability. Moreover, the fabrication process involves multiple steps of lithography, film deposition, and etching to form the gratings, the spacer layer, and the QWPs and therefore must be systematically designed to avoid structural integration issues, such as etching damage, poor film adhesion, high surface roughness, and feature distortion. Figure $3 \mathrm{a}$ illustrates the fabrication procedures we used to demonstrate the ODLM structures. First, gold nanogratings were fabricated on fused silica substrates by electronbeam lithography (EBL), metal evaporation, and metal liftoff. Then, a dielectric spacer layer $(350 \mathrm{~nm})$ of $\mathrm{SiO}_{x}$ was sputtered to cover the nanogratings, followed by plasmaenhanced chemical vapor deposition (PECVD) of $\alpha$-silicon $(\alpha-\mathrm{Si})$. The $\alpha$-Si layer was patterned into nanopillars by EBL and inductively coupled plasma reactive-ion etching (ICP RIE) using an $\mathrm{SiO}_{x}$ hard mask. The detailed fabrication steps are presented in the "Materials and methods" section and Supplementary Information (Fig. S3). Figure 3b shows a scanning microscope (SEM) image of the fabricated polarizing nanograting composed of 120 -nm-thick gold nanogratings with a $230 \mathrm{~nm}$ period and $50 \%$ duty cycle. The nanogratings demonstrated were thinner than the optimized designs due to challenges in fabricating thicker nanogratings with low defect density. The use of metallic nanogratings allows a high LPER but also poses certain integration challenges because of instability of metal under elevated temperatures, poor adhesion to dielectrics, and rough surface. Here, we demonstrated that the use of a sputtered $\mathrm{SiO}_{x}$ dielectric spacer layer resolves or mitigates these problems. The nanograting samples were baked at a moderate temperature to remove the surface water molecules and in situ sputter-cleaned to remove the contaminants on the surface immediately before a room temperature sputtering process to cover the nanogratings with an $\mathrm{SiO}_{x}$ dielectric layer. The sputtered $\mathrm{SiO}_{x}$ spacer layer exhibited excellent adhesion to the fused silica substrate, filled the trenches in the nanogratings ${ }^{48}$, and greatly reduced the surface roughness resulting from the embedded nanogratings. As inspected by cross-sectional SEM (Fig. 3d) and atomic force microscopy (Fig. 3e, f), sputtering a $350 \mathrm{~nm}$ silicon oxide spacer layer onto the nanograting surface significantly decreased the surface roughness $(\mathrm{Ra}=11.5 \mathrm{~nm}$, as shown in Fig. 3e, f). The reduced surface roughness was crucial to the subsequent fabrication of $\mathrm{Si}$ nanopillar QWPs. Fourier transform infrared spectroscopy (FTIR) measurements and full-wave simulation results show that the $\mathrm{SiO}_{x}$-coated nanogratings experience only a slight degradation of the LPER (Supplementary Information Fig. S4), indicating that the dielectric layer fabrication process only minorly impacted the nanograting performance. In addition to the structural importance of the $\mathrm{SiO}_{x}$ spacer, the spacer helps to minimize the near-field interactions between the nanogratings and QWPs and ensure reliable device operation. Full-wave simulation shows that the optimal thickness is between 300 and $400 \mathrm{~nm}$ for our design in the 1.3-1.5 $\mu \mathrm{m}$ wavelength range (Supplementary Information Fig. S5).

Finally, the Si metasurface QWPs were aligned on top of the polarizing nanogratings. The metasurface QWP is composed of densely packed silicon nanopillars, as shown in Fig. 3c (length $480 \mathrm{~nm}$, width $160 \mathrm{~nm}$, and thickness $700 \mathrm{~nm})$. The nanoscale dimensions and large height-width aspect ratios (HWAR $>4$ ) pose significant challenges in fabrication. We used a double-layer hard mask ( $60 \mathrm{~nm} \mathrm{Cr}$ on top of $160 \mathrm{~nm}$ of $\mathrm{SiO}_{x}$ ) to pattern the silicon nanopillars with the nanoscale geometries required. A 60-nm-thick $\mathrm{Cr}$ mask fabricated by EBL and metal liftoff was used to pattern the $\mathrm{SiO}_{x}$ mask. Then, the $\mathrm{SiO}_{x}$ layer served as a second hard mask for ICP RIE etching of $\alpha$-Si nanopillars with a large HWAR. More details are provided in the "Materials and methods" section. Noticeably, our optimized fabrication process enabled etching of the nanopillars with steep sidewalls $\left(<8^{\circ}\right.$ tilt angle, Supplementary Information Fig. S6), a lateral pillar gap as small as $200 \mathrm{~nm}$, and an HWAR > 4 (Supplementary Information Fig. S3). To consider the sidewall inclination angle of the silicon nanopillar, we modified the structure dimensions in the numerical 


\section{a}
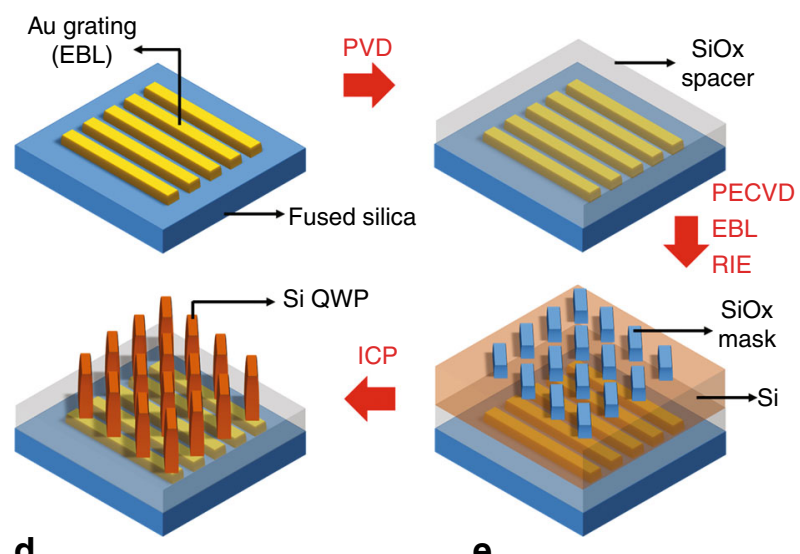

d

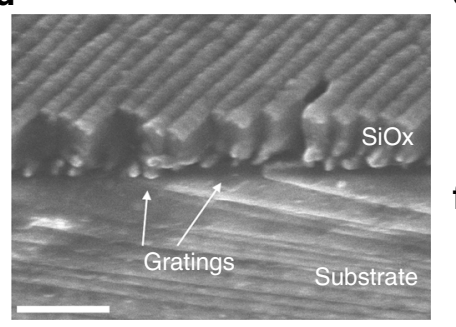

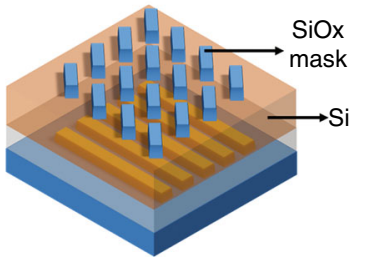

e

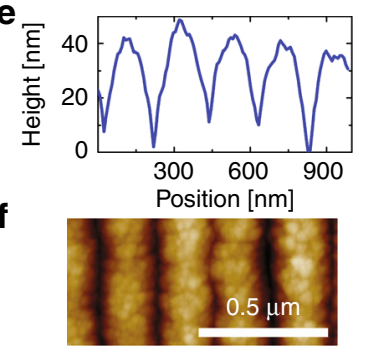

b
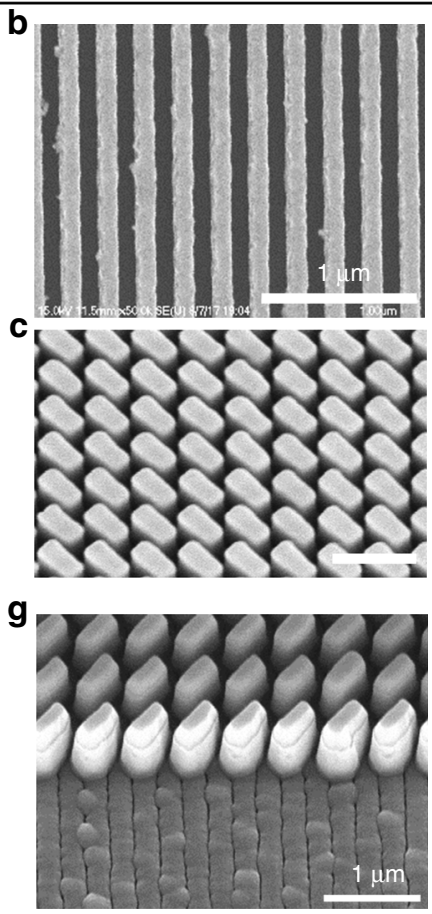

Fig. 3 Fabrication of ODLM-based CPL filters. a Schematic illustration of major fabrication steps. $\mathbf{b}$ Scanning electron microscope (SEM) image of the nanograting before QWP metasurface integration. c SEM image of silicon QWP. $\mathbf{d}$ Cross section of grating covered by fused silica. The fringes on the surface of the $\mathrm{SiO}_{x}$ spacer layer reflect the underlying grating thickness and duty cycle. e, $\mathbf{f}$ Atomic force microscope (AFM) images of the nanograting after PVD deposition of $\mathrm{SiO}_{x}(\sim 350 \mathrm{~nm})$, indicating mitigated surface roughness (Ra $\left.=11.5 \mathrm{~nm}\right)$. $\mathbf{g}$ Tilted-view SEM image of ODLM structure. The bright thin layer on top of each Si QWP pillar corresponds to residual $\mathrm{SiO}_{x}$ mask. All the SEM scale bars are $1 \mu \mathrm{m}$, and the AFM scale bar is $0.5 \mu \mathrm{m}$

model and performed structure optimization accordingly to determine the device geometries (see Supplementary Information Fig. S7). Our theoretical analysis suggests that an inclination angle from $0^{\circ}$ to $10^{\circ}$ can achieve high CPERs (>300) at any desired operational wavelength.

\section{Device characterization and discussion}

The performance of the metasurface QWPs and linear polarizing nanogratings were first characterized using FTIR (see "Materials and methods" section). To characterize the metasurface QWPs, LP light was directed incident to the device under testing with the electric field vector oriented at $45^{\circ}$ with respect to the slow $(U-)$ axis of the QWP. The state of polarization (SoP) of the transmitted light was measured with a rotating linear polarizer (polarization analyzer (PA)) to extract the phase retardation. The phase retardation and transmission spectra measured for the metasurface QWP designed within the telecom wavelength bands are shown in Fig. 4a. At the operation wavelength $(1.47 \mu \mathrm{m})$, the phase retardation between the fast $(V-)$ and slow $(U-)$ axes is exactly $\pi / 2$, and the transmission efficiency of the QWP is close to $90 \%$. The nanograting linear polarizer fabricated (width 120, height 122, and period $230 \mathrm{~nm}$ as fabricated) exhibited a LPER of 40-50 and transmission efficiency $>90$ at wavelengths of $\sim 1.5 \mu \mathrm{m}$ (Fig. 4b). We attribute the lower LPER mainly to the nanograting thickness $(120 \mathrm{~nm})$ being smaller than that of the optimized device designs $(190 \mathrm{~nm})$ (see Fig. S2a). The chip-integrated ODLM-based CPL polarization filters were characterized with both LCP and RCP input light, as illustrated in the schematic in Fig. 4c (see "Materials and methods" section). Figure 4c shows the transmission spectra measured for three devices fabricated on the same substrate for both LCP and RCP input light in proximity to the operation wavelengths of the devices. These devices were designed for different operation wavelengths from 1.4 to $1.6 \mu \mathrm{m}$. The devices all have high transmission efficiency (70-80\%) for LCP light and minimal transmission (2-3\%) for RCP light. The maximum CPERs (defined as $I_{\mathrm{LCP}} / I_{\mathrm{RCP}}$ ) for all three devices are over 30, as shown in Fig. 4d. All of the devices provide CPERs of more than 10 over a wavelength range of $150 \mathrm{~nm}$ at approximately the operation wavelength. Compared with other on-chip solid-state-based CPL filters reported in the literature, the ODLM-based CPL filters provide the best performance when taking both CPER and efficiency in consideration (see Supplementary Information Table S1).

Compared with the simulation results of the optimized device design, there is still much room for improvement of the CPERs of the ODLM-based CPL filters. Due to the 


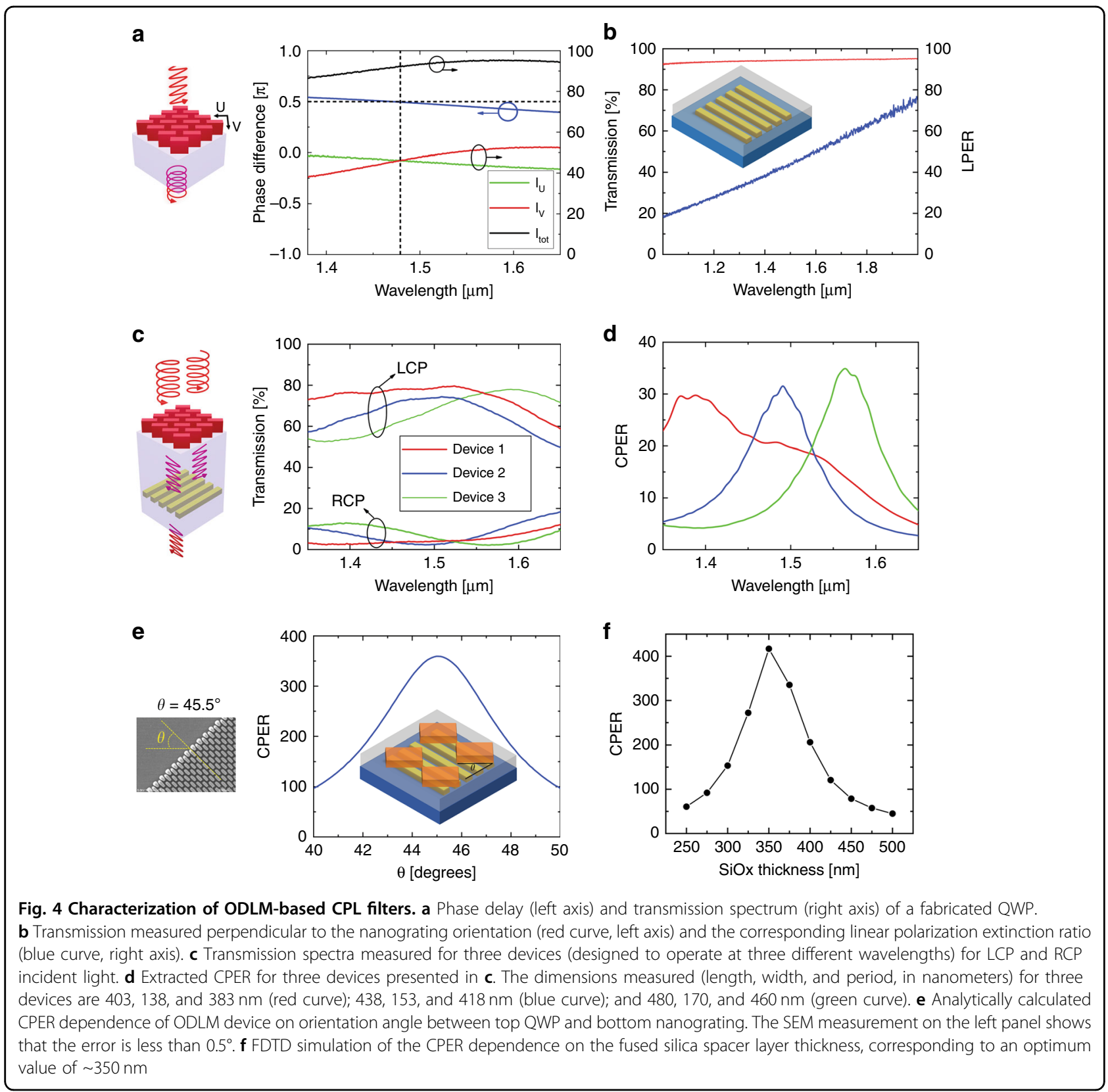

limitations of the nanofabrication process, the devices experimentally demonstrated deviation from the simulations in a number of aspects. In addition to the imperfections of the fabricated nanogratings and silicon metasurfaces discussed previously, the alignment errors between the metasurfaces and the nanogratings underneath affect the device performance. Figure 4e shows the dependence of the CPER on the error in the rotational alignment between the slow axis of the metasurface QWP and the LP polarizer. From the SEM images of the samples fabricated, we estimated a degree error of less than 0.5 from the ideal value $\left(45^{\circ}\right)$ (Supplementary Information Fig. S8). According to the results shown in Fig. 4e, this angle deviation leads to a slight decrease $(<6 \%)$ in the CPER of the CP filter. Further, the translational displacement between the silicon pillars in the QWP and the nanowires in the linear polarizer only slightly affected the performance of the ODLM structure design $(<2 \%$, Supplementary Information Fig. S9). In addition, we studied the dependence of the CPER on the dielectric spacer layer thickness at the working wavelength (Fig. 4f) and found a reasonably small degradation of the CPER $(<5 \%)$ for a deviation of $5 \mathrm{~nm}$ from the spacer thickness designed $(350 \mathrm{~nm})$. Based on the experimental evidence and theoretical analysis, we conclude that the key practical limitations in the double-layer integration do not significantly 


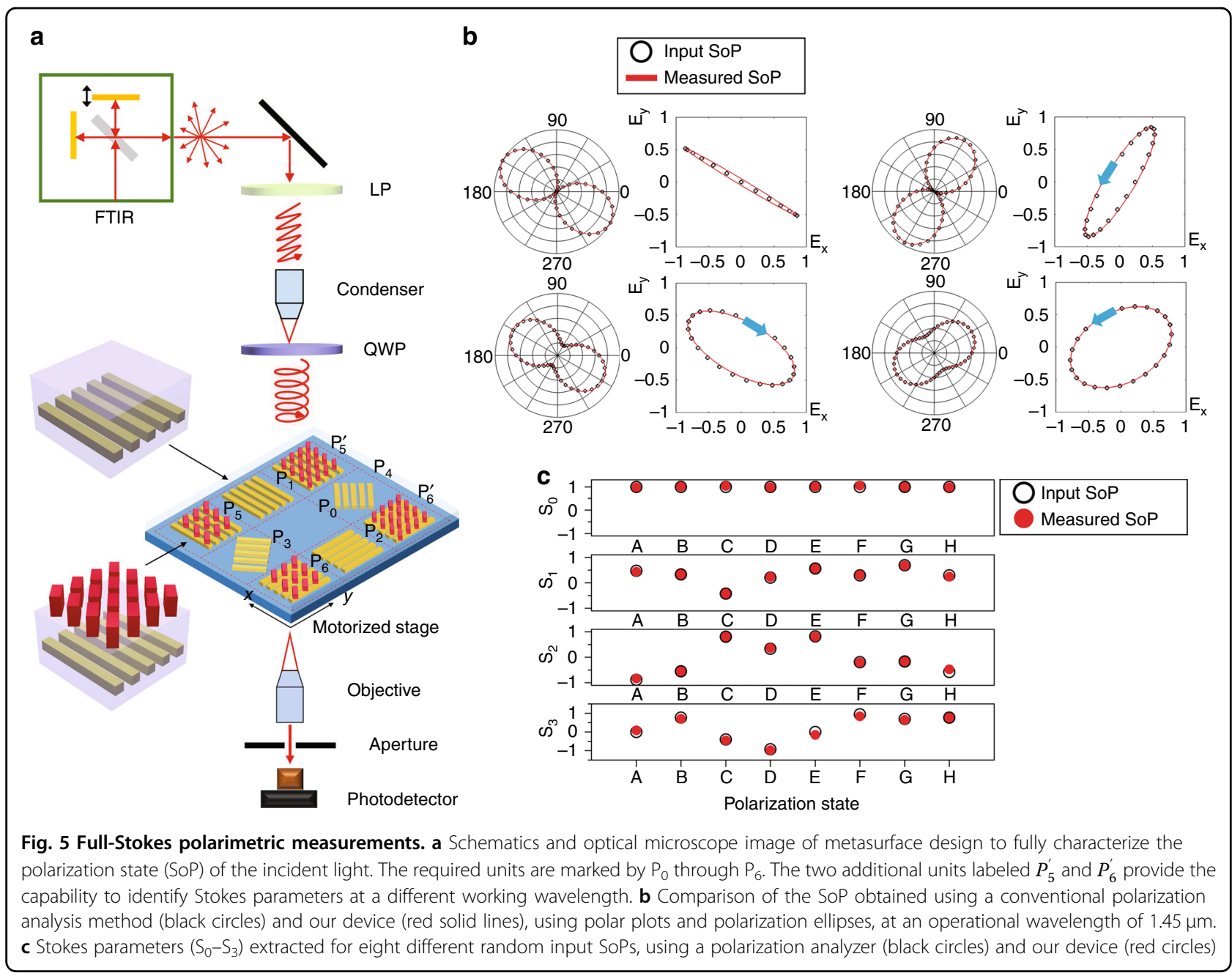

affect the CPERs of the ODLM-based CPL filters fabricated. However, significant improvement of the device performance could be achieved by improving the LPER of the polarizing nanogratings. Our numerical simulations reveal that a change in the nanograting thickness from 120 to $\sim 190 \mathrm{~nm}$ increases the LPER from $\sim 50$ to over 400 .

To demonstrate the concept of on-chip polarimetric detection, we integrated an array of polarization filters on the same chip, including four LP filters and two ODLMbased CP filters, for full-Stokes polarization state measurements based on a spatial division scheme ${ }^{8}$. The LP filters $\left(\mathrm{P}_{1}, \mathrm{P}_{2}, \mathrm{P}_{3}\right.$, and $\left.\mathrm{P}_{4}\right)$ are simply nanowire gratings oriented in four different orientations, $0,90,-45$, and $45^{\circ}$, with respect to the $x$-axis (Fig. $5 \mathrm{a}$ ). The two CP filters, $\mathrm{P}_{5}$ and $\mathrm{P}_{6}$, are selectively transmitting only LCP and RCP light, respectively $\left(P_{5}^{\prime}\right.$ and $P_{6}^{\prime}$ are backup structures for $P_{5}$ and $\left.P_{6}\right)$. An empty cell $P_{0}$ without any filters is located in the center to measure the total light intensity $\mathrm{I}_{0}$. All the filters and the empty cells have the same area of $80 \mu \mathrm{m} \times$ $80 \mu \mathrm{m}$. The incident light is filtered with these six spatially distributed micrometer-size polarization filters and then measured by separately photodetectors to obtain the polarized components. Given the light transmitted by $\mathrm{P}_{i}$, denoted $\mathrm{I}_{i}(i=0-6)$, the Stokes parameters, $\left(\mathrm{S}_{0}, \mathrm{~S}_{1}, \mathrm{~S}_{2}\right.$, and $S_{3}$ ), of the incident light are then calculated as follows: ${ }^{8}$

$$
\left\{\begin{array}{l}
S_{0}=I_{0} \\
S_{1}=I_{2}-I_{1} \\
S_{2}=I_{4}-I_{3} \\
S_{3}=I_{6}-I_{5}
\end{array}\right.
$$

Notably, this design can be used to detect the SoP of any incident light, including partially polarized light. When the incident light is fully polarized, $\mathrm{I}_{0}{ }^{2}=\mathrm{S}_{1}{ }^{2}+\mathrm{S}_{2}{ }^{2}+\mathrm{S}_{3}{ }^{2}$, while for partially polarized light, $\mathrm{I}_{0}{ }^{2}>\mathrm{S}_{1}{ }^{2}+\mathrm{S}_{2}{ }^{2}+\mathrm{S}_{3}{ }^{2}$.

To test the performance of the microscale polarization filter array fabricated, we used FTIR with an infrared microscope to build the measurement setup, as shown in Fig. 5a. Unpolarized light from the FTIR internal source was transmitted through a conventional linear polarizer and tuneable LC waveplate, separated by a reflective objective (also known as a condenser), to generate an 
arbitrary SoP. The reflective objective was used to focus the light onto the device, which was placed on a motorized stage. By placing the tuneable LC waveplate between the objective and the device under testing, we were able to minimize the impact of the reflective objective on the SoP of the light incident on the device. The light transmitted through the sample was then collected by an infrared objective. An aperture was used at the image plane to select the measurement region $\left(30 \times 30 \mu \mathrm{m}^{2}\right)$ on the device. Such a scanning imaging system allows us to characterize the light transmitted through all seven cells by simply changing the lateral displacement of the motorized stage. For calibration purposes, we removed the sample and used a rotating PA to characterize the SoP of the incident light (see "Materials and methods" section for more details). Figure $5 \mathrm{~b}$ presents the polar plots for four representative SoPs. The measured results obtained with the on-chip polarimeter (red) are consistent with those obtained from the PA (black). We extracted the ellipse plots for all the SoPs to evaluate the accuracy of the orientation and ellipticity angle measurements. Based on all the measurement results of eight SoPs (see "results" for the other SoPs in Supplementary Information, Fig. S11), the average errors of the orientation and ellipticity angles are $0.046^{\circ}$ and $2.21^{\circ}$, respectively. Moreover, the handedness of the light can be obtained with our technique, as shown on the ellipse plots labeled with blue arrows, which is not available from the measurements taken by the rotating polarizer. Figure $5 \mathrm{c}$ shows a summary of the Stokes parameters $\left(S_{0}, S_{1}, S_{2}\right.$, and $\left.S_{3}\right)$ obtained by our device (all the Stokes parameters were normalized by $\mathrm{S}_{0}$ ). To evaluate the accuracy of the measurements, we characterized each SoP with the rotating PA and the extracted corresponding Stokes parameters (shown in Fig. 5c). The average measurement errors of $S_{1}, S_{2}$, and $S_{3}$ were $1.9 \%$, $2.7 \%$, and $7.2 \%$, respectively. Based on the Stokes parameters measured, the measurement errors for the degree of LP $\left(\sqrt{S_{1}^{2}+S_{2}^{2}} / S_{0}\right)$ and degree of circular polarization $\left(S_{3} / S_{0}\right)$ are $3.3 \%$ and $7.9 \%$, respectively. Compared with other plasmonic or dielectric metasurface-based polarimetric detection techniques reported in the literature, our devices exhibit the best performances in measurement accuracy and optical efficiency (Table 1). Even though we used a scanning imaging system to perform the device characterization in our experiment, a spatial division measurement scheme can be employed to perform fullStokes polarization detection in one snapshot without moving parts. In such a configuration, the polarization filter array is directly integrated on the top of a photodetector array so that the incident light filtered by each microscale polarization filter is measured by the corresponding photodetector beneath that filter. Thus, all the polarization components can be obtained by a photodetector array integrated with microscale polarization filters.

\section{Discussion}

Here, we reported bioinspired chiral double-layer metasurface structures (ODLMs) with both strong chiral optical effects and high optical efficiencies. Based on these structures, we have experimentally demonstrated submicron-thick CP light filters with CPER as high as 35 and maximum transmission efficiency close to $80 \%$. The best operational wavelengths can be tailored by varying the design parameters from 1.3 to $1.6 \mu \mathrm{m}$. We attribute the high transmission efficiency and extinction ratio to the low insertion loss of the dielectric metasurface QWP and the nanowire linear polarizer, the high accuracy of the phase delay of the metasurface QWP, and the high LPER of the nanowire linear polarizer. The proposed ODLMbased $\mathrm{CP}$ filers can be very compact, with thickness less than $1 \mu \mathrm{m}$ and minimal lateral dimensions of $1 \times 1 \mu \mathrm{m}^{2}$ (to maintain a CPER greater than 20 and transmission efficiency $>60 \%$; see Fig. S13). Our experimental and theoretical analyses show that the device performance could be further enhanced by improving the fabrication procedures and improving the performance and bandwidth of the metasurface QWP ${ }^{49}$. We also monolithically integrated the ODLM-based CP filers with LP nanograting filters for full-Stokes polarization measurements of input light with arbitrary polarization states. Our polarimetric detection devices demonstrate the best performance in measurement accuracy and optical efficiency (80\%). In addition to their superior performance, the chiral metasurface structures and polarimetric devices presented exhibit various advantages, such as easy on-chip integration, ultracompact footprint, manufacturing scalability, and broad wavelength range. Therefore, our work holds great promise for chip-integrated systems for quantum-based optical computing and information processing, CD spectroscopy, polarimetric imaging, and sensing applications.

\section{Materials and methods}

\section{Numerical simulations}

The FDTD simulations were performed using Lumerical Inc. FDTD solver. The material optical properties of the $\alpha$-Si and silicon oxide were determined from ellipsometry measurements using a J.A. WOOLLAM system. The residual $\mathrm{SiO}_{x}$ mask layer and sidewall tilting were considered in accordance with the SEM results. Here, we simulated one unit cell with normal plane wave source(s) incidence, the in-plane boundary conditions were periodic, and perfectly matched layers were used for the outof-plane boundaries. CP light was simulated by a superposition of two linearly polarized sources with $\pi / 2$ relative phase retardance. We set a refined mesh with a minimum mesh size of $5 \mathrm{~nm}$ for silicon nanopillars. We confirmed that all the simulations converged at an auto-shutoff value of $10^{-5}$. 


\section{Fabrication}

(1) Gold polarizing nanograting: The fused silica substrate was spin coated with double-layer poly (methyl methacrylate) (PMMA) (170 nm, $495 \mathrm{k}$ followed with $35 \mathrm{~nm}, 950 \mathrm{k}$ ) and a very thin $(\sim 5 \mathrm{~nm})$ thermally evaporated $\mathrm{Cr}$ layer for charge dissipation. Then, the samples were exposed by ebeam lithography (EBL, JEOL JBX-6000FS) and developed in a mixture of methyl isobutyl ketone (MIBK) and isopropanol (IPA) at a ratio 1:3. The sample was cleaned by oxygen plasma (PlasmaTherm 790, 5 sccm O2 with 8 mTorr chamber pressure, $20 \mathrm{~W}$ ) for a few seconds to remove the residual PMMA on the exposed region. Then, a $4 \mathrm{~nm}$ adhesive $C r$ layer followed by $122 \mathrm{~nm}$ of gold was deposited by thermal evaporation (Edwards Auto 306) without breaking vacuum. Subsequently, the $\mathrm{Au} / \mathrm{Cr}$ nanogratings were lifted off by soaking the sample in acetone for more than $12 \mathrm{~h}$ followed by sonication for $1 \mathrm{~min}$.

(2) Spacer deposition: A 5-min oxygen plasma etch was applied to the samples to completely remove organic contaminants. Then, the samples were immediately placed in the sputtering chamber (Lesker PVD 75) and covered with a $350 \mathrm{~nm} \mathrm{SiO}_{\mathrm{x}}$ spacer layer (RF power $250 \mathrm{~W})$ at a rate of $0.6 \AA / \mathrm{s}$.

(3) Si QWP fabrication: Amorphous silicon $(\alpha-\mathrm{Si})$ of $700 \mathrm{~nm}$ was deposited by PECVD (Oxford Plasmalab $\left.100,350^{\circ} \mathrm{C} / 15 \mathrm{~W}\right)$ on the $\mathrm{SiO}_{\mathrm{x}}$ spacer layer, followed by deposition of $160 \mathrm{~nm} \mathrm{SiO}_{\mathrm{x}}\left(350^{\circ}\right.$ $\mathrm{C} / 20 \mathrm{~W}$ ) without breaking vacuum as a hard mask layer. Another $10 \mathrm{~nm} \mathrm{Cr}$ layer was deposited onto the sample surface to avoid charging effects during EBL fabrication. Again, a double-layer PMMA resist $(120 \mathrm{~nm}, 495 \mathrm{k}$ followed with $50 \mathrm{~nm}, 950 \mathrm{k}$ ) was coated on the sample, exposed with EBL, and developed in IPA/MIBK as described earlier. Then, $60 \mathrm{~nm}$ of $\mathrm{Cr}$ was thermally evaporated onto the sample and lifted off in acetone as described earlier to form the $\mathrm{Cr}$ hard mask. Next, the $10 \mathrm{~nm} \mathrm{Cr}$ adhesion layer was etched through by ICP etching (Advanced Vacuum Apex SLR, $\mathrm{Cl}_{2} / \mathrm{O}_{2} 22 / 8 \mathrm{sccm}$, $20 \mathrm{mTorr}$ ICP/ bias power $400 / 100 \mathrm{~W}$ ) to form isolated $\mathrm{Cr}$ nanostructure masks, which further masked the anisotropic etching of the $\mathrm{SiO}_{\mathrm{x}}$ hard mask by RIE (Plasmatherm RIE 790, $\mathrm{CHF}_{3} / \mathrm{O}_{2} 40$ / $3 \mathrm{sccm}, 40 \mathrm{mTorr}, 250 \mathrm{~W})$ and stopped at the $\alpha-\mathrm{Si}$ layer. Then, the $\mathrm{Cr}$ was removed by $\mathrm{CR}-4 \mathrm{~s}$ (Cyantek) etchant, and the $\alpha$-Si layer was etched by ICP RIE (ICP/bias power of 250/140 W, 10 mTorr, $\mathrm{Cl}_{2}: \mathrm{Ar}=100 / 5 \mathrm{sccm}$ ) using the $\mathrm{SiO}_{\mathrm{x}}$ mask to complete the device fabrication.

\section{Measurements}

The optical transmission measurements were performed using a Bruker Vertex 70 FTIR spectrometer connected to a Hyperion 2000 mid-IR microscope (Fig. S10). For the QWP measurements, we used one linear polarizer in the optical path immediately in front of the sample under testing to ensure LP incidence. Another linear polarizer was placed in the optical path immediately behind the sample to analyze the polarization state of the output light by rotating the polarizer and measuring the transmitted light with an IR photodetector. Two 15x objective and condenser lenses with N.A. $=0.4$ were used. For CP detection measurements and full-Stokes parameter measurements, we used one linear polarizer and an LC waveplate (LCC1223-C by Thorlabs, Inc.) to generate CP light with both handedness and arbitrary polarization state that was incident to the device under testing. As a calibration process, we characterized the polarization state of the incident light with a rotating linear polarizer and QWP. All the transmission spectra were normalized with respect to that of the bare fused silica substrate to eliminate the impact of the substrate.

\section{Acknowledgements \\ This research was supported in part by the AFOSR YIP under grant no. FA9550-16-1-0183, the National Science Foundation under grant no. 1809997, and Arizona State University startup funds provided to Y.Y. C.W. and X.C. acknowledge partial support from the National Science Foundation under grant no. 1711412 and Arizona State University startup funds provided to C.W. The devices were fabricated in the NanoFab and Eyring Materials Center (EMC) at Arizona State University. Access to the NanoFab and/or EMC was supported, in part, by NSF contract ECCS-1542160.}

\section{Author details}

${ }^{1}$ School of Electrical, Computer and Energy Engineering, Arizona State University, Tempe, AZ 85281, USA. ${ }^{2}$ Centre for Photonic Innovation, Arizona State University, Tempe, AZ 85281, USA. ${ }^{3}$ Biodesign Centre for Molecular Design \& Biomimetics, Arizona State University, Tempe, AZ 85281, USA

\section{Author contributions}

Y.Y. conceived the idea. Y.Y. and C.W. supervised the study. A.B., X.C., and J.B. performed device design and fabrication. A.B., J.C., P.A., and Z.H. supported the thin film growth and characterization. A.B. and J.B. carried out the measurements and data analysis. A.B., Y.Y. and C.W. wrote the manuscript, and all authors contributed to the manuscript.

\section{Conflict of interest}

The authors declare that they have no conflict of interest.

Supplementary information is available for this paper at https://doi.org/ 10.1038/s41377-019-0184-4.

Received: 21 February 2019 Revised: 1 July 2019 Accepted: 24 July 2019 Published online: 28 August 2019

\footnotetext{
References

1. Farshchi, R. et al. Optical communication of spin information between light emitting diodes. Appl. Phys. Lett. 98, 162508 (2011).

2. Sherson, J. F. et al. Quantum teleportation between light and matter. Nature 443, 557-560 (2006).
} 
3. Wagenknecht, $C$. et al. Experimental demonstration of a heralded entanglement source. Nat. Photonics 4, 549-552 (2010).

4. Ranjbar, B. \& Gill, P. Circular dichroism techniques: biomolecular and nanostructural analyses-a review. Chem. Biol. Drug Des. 74, 101-120 (2009).

5. Martinez, N. J. D. et al. Single photon detection in a waveguide-coupled Geon-Si lateral avalanche photodiode. Opt. Express 25, 16130-16139 (2017).

6. Giakos, G. C. Multifusion, multispectral, optical polarimetric imaging sensing principles. IEEE Trans. Instrum. Meas. 55, 1628-1633 (2006).

7. Garcia, N. M. et al. Surface normal reconstruction using circularly polarized light. Opt. Express 23, 14391-14406 (2015).

8. Berry, H. G., Gabrielse, G. \& Livingston, A. E. Measurement of the Stokes parameters of light. Appl. Opt. 16, 3200-3205 (1977).

9. Vedel, M., Breugnot, S. \& Lechocinski, N. Full Stokes polarization imaging camera. In Proc. of the SPIE 8160, Polarization: Science and Remote Sensing V. 8160X (SPIE, San Diego, CA, USA, 2011).

10. De Martino, A. et al. Optimized mueller polarimeter with liquid crystals. Opt. Lett. 28, 616-618 (2003).

11. Sánchez-Carnerero, E. M. et al. Circularly polarized luminescence by visiblelight absorption in a chiral O-BODIPY dye: unprecedented design of CPL organic molecules from achiral chromophores. J. Am. Chem. Soc. 136, 3346-3349 (2014).

12. Yang, $Y$. et al. Circularly polarized light detection by a chiral organic semiconductor transistor. Nat. Photonics 7, 634-638 (2013).

13. Hu, J. P. et al. All-dielectric metasurface circular dichroism waveplate. Sci. Rep. 7 srep41893 (2017).

14. Dong, J. F. et al. Bi-layer cross chiral structure with strong optical activity and negative refractive index. Opt. Express 17, 14172-14179 (2009).

15. Zhao, Y. et al. Chirality detection of enantiomers using twisted optical metamaterials. Nat. Commun. 8, 14180 (2017).

16. Decker, M. et al. Circular dichroism of planar chiral magnetic metamaterials. Opt. Lett. 32, 856-858 (2007).

17. Li, W. et al. Circularly polarized light detection with hot electrons in chiral plasmonic metamaterials. Nat. Commun. 6, 8379 (2015).

18. Chen, W. B. et al. Experimental confirmation of miniature spiral plasmonic lens as a circular polarization analyzer. Nano Lett. 10, 2075-2079 (2010).

19. Zhu, A. Y. et al. Giant intrinsic chiro-optical activity in planar dielectric nanostructures. Light.: Sci. Appl. 7, 17158 (2018).

20. Gansel, J. K. et al. Gold helix photonic metamaterial as broadband circular polarizer. Science 325, 1513-1515 (2009).

21. Frank, B. et al. Large-area 3D chiral plasmonic structures. ACS Nano 7, 6321-6329 (2013).

22. Zhang, M. L. et al. Nanoimprinted chiral plasmonic substrates with threedimensional nanostructures. Nano Lett. 18, 7389-7394 (2018).

23. Zhao, Y., Belkin, M. A. \& Alù, A. Twisted optical metamaterials for planarized ultrathin broadband circular polarizers. Nat. Commun. 3, 870 (2012).

24. Kuzyk, A. et al. DNA-based self-assembly of chiral plasmonic nanostructures with tailored optical response. Nature 483, 311-314 (2012).

25. Fan, Z. Y. \& Govorov, A. O. Plasmonic circular dichroism of chiral metal nanoparticle assemblies. Nano Lett. 10, 2580-2587 (2010).

26. Shen, X. B. et al. Three-dimensional plasmonic chiral tetramers assembled by DNA origami. Nano Lett. 13, 2128-2133 (2013).
27. Zhao, X. H. et al. Circle polarization shift keying with direct detection for freespace optical communication. J. Opt. Commun. Netw. 1, 307-312 (2009).

28. Han, Y. \& Li, G. F. Coherent optical communication using polarization multipleinput-multiple-output. Opt. Express 13, 7527-7534 (2005).

29. Pust, N. J. \& Shaw, J. A. Digital all-sky polarization imaging of partly cloudy skies. Appl. Opt. 47, H190-H198 (2008).

30. Snik, F. et al. An overview of polarimetric sensing techniques and technology with applications to different research fields. In Proc. of the SPIE 9099, Polarization: Measurement, Analysis, and Remote Sensing XI. 90990B (SPIE, Baltimore, MA, USA, 2014).

31. Kunnen, B. et al. Application of circularly polarized light for non-invasive diagnosis of cancerous tissues and turbid tissue-like scattering media. J. Biophotonics 8, 317-323 (2015).

32. Salomatina-Motts, E., Neel, V. A. \& Yaroslavskaya, A. N. Multimodal polarization system for imaging skin cancer. Opt. Spectrosc. 107, 884-890 (2009).

33. Patel, R. et al. Polarization-sensitive multimodal imaging for detecting breast cancer. Cancer Res. 74, 4685-4693 (2014).

34. Pors, A., Nielsen, M. G. \& Bozhevolnyi, S. I. Plasmonic metagratings for simultaneous determination of Stokes parameters. Optica 2, 716-723 (2015).

35. Chen, W. T. et al. Integrated plasmonic metasurfaces for spectropolarimetry. Nanotechnology 27, 224002 (2016).

36. Jung, $M$. et al. Polarimetry using graphene-integrated anisotropic metasurfaces. ACS Photonics 5, 4283-4288 (2018).

37. Mueller, J. P. B., Leosson, K. \& Capasso, F. Ultracompact metasurface in-line polarimeter. Optica 3, 42-47 (2016).

38. Lee, K. et al. Ultracompact broadband plasmonic polarimeter. Laser Photonics Rev. 12, 1700297 (2018).

39. Afshinmanesh, F. et al. Measurement of the polarization state of light using an integrated plasmonic polarimeter. Nanophotonics 1, 125-129 (2012).

40. Arbabi, E. et al. Full-Stokes imaging polarimetry using dielectric metasurfaces. ACS Photonics 5, 3132-3140 (2018).

41. Chiou, T. H. et al. Circular polarization vision in a stomatopod crustacean. Curr. Biol. 18, 429-434 (2008)

42. Daly, I. M. et al. Dynamic polarization vision in mantis shrimps. Nat. Commun. 7, 12140 (2016).

43. Land, M. F. \& Osorio, D. Extraordinary color vision. Science 343, 381-382 (2014).

44. Li, Z. C. et al. Realizing broadband and invertible linear-to-circular polarization converter with ultrathin single-layer metasurface. Sci. Rep. 5, 18106 (2015).

45. Khorasaninejad, M. et al. Metalenses at visible wavelengths: diffraction-limited focusing and subwavelength resolution imaging. Science 352, 1190-1194 (2016).

46. Khorasaninejad, M. \& Crozier, K. B. Silicon nanofin grating as a miniature chirality-distinguishing beam-splitter. Nat. Commun. 5, 5386 (2014).

47. Zheng, G. X. et al. Metasurface holograms reaching $80 \%$ efficiency. Nat. Nanotechnol. 10, 308-312 (2015).

48. Cao, H. et al. Fabrication of $10 \mathrm{~nm}$ enclosed nanofluidic channels. Appl. Phys. Lett. 81, 174-176 (2002).

49. Kikuta, H., Ohira, Y. \& Iwata, K. Achromatic quarter-wave plates using the dispersion of form birefringence. Appl. Opt. 36, 1566-1572 (1997).

50. Espinosa-Soria, A. et al. On-chip optimal Stokes nanopolarimetry based on spin-orbit interaction of light. Nano Lett. 17, 3139-3144 (2017). 